\section{The Authors' Reply}

\section{Dear Editor,}

In response to our article, ${ }^{[1]}$ van Luijn questions whether there is a role for economic evaluation in decision making about coverage or payment for new medicines. Our answer: yes, economic evaluations can provide information about the efficiency of pharmaceuticals and nondrug technologies that can help decision makers, including insurers and payers, decide about the best use of healthcare resources. In effect, evidence suggests increased use of economic evaluation information, complementary to the information from clinical studies and other evaluative approaches such as budget impact analyses, in decision making. ${ }^{[2,3]}$

Performing economic evaluations requires decision makers, manufacturers, providers and others involved in evidence and decision-making processes to invest effort in collecting and synthesizing data on the effect of technologies on outcomes and costs. As relevant data may be readily available from existing research, these efforts need not be demanding or costly. This and the fact that using economic evaluation information in making decisions can improve health and healthcare make investments in economic evaluations likely cost effective.

The findings of our study reinforce the need for economic evaluations to be methodologically sound so that consequent coverage or payment decisions are credible and appropriate. To enhance the use of economic evaluation in specific circumstances or decision contexts, for example, when only indirect evidence exists on health technologies, or when heterogeneity is expected in outcomes and patient groups, methods for evaluation will continue to be advanced. If any such undertaking is done with consideration to the needs of decision makers and others, it would be more likely that economic evaluations are found useful and are applied in decision-making practice.

Ties Hoomans, ${ }^{1}$ Johan L. Severens, ${ }^{2}$ Nicole van der Roer ${ }^{3}$ and Gepke O. Delwel ${ }^{3}$

1 Section of Hospital Medicine, Department of Medicine, University of Chicago, Chicago, IL, USA

2 Institute of Health Policy and Management, Erasmus University Rotterdam, Rotterdam, the Netherlands

3 Diemen, the Netherlands

\section{Acknowledgements}

The views expressed in this letter are those of the authors and should not be attributed to the authors' employers. The authors have no conflicts of interest that are directly relevant to the content of this letter.

\section{References}

1. Hoomans T, Severens JL, van der Roer N, et al. Methodological quality of economic evaluations of new pharmaceuticals in the Netherlands. Pharmacoeconomics 2012; 30 (3): 219-27

2. Galani C, Rutten FF. Self-reported healthcare decisionmakers' attitudes towards economic evaluations of medical technologies. Curr Med Res Opin 2008; 24 (11): 3049-58

3. Brousselle A, Lessard C. Economic evaluation to inform health care decision-making: promise, pitfalls and a proposal for an alternative path. Soc Sci Med 2011; 72 (6): 832-9 